\title{
Mycological Profile of Respiratory Tract Samples in a Tertiary Care Hospital from Western India
}

\author{
Athira Jayaram ${ }^{1}$, Khushboo Sareen ${ }^{2}$, Ashiwini Dedwal ${ }^{3}$, Sushma Pednekar ${ }^{4}$, \\ Sunil Bhamare $^{5}$, Swati Mulshingkar ${ }^{6}$, Rajesh Karyakarte ${ }^{7}$ \\ ${ }^{1,2}$ PG Student, Department of Microbiology, BJGMC, Pune. \\ ${ }^{3}$ Assistant Professor, MD Microbiology, Department of Microbiology, BJGMC, Pune \\ 4,5,6 Associative Professor, MD Microbiology, Department of Microbiology, BJGMC, Pune \\ ${ }^{7} \mathrm{HOD}$ and Professor, MD Microbiology, Department of Microbiology, BJGMC, Pune
}

Corresponding Author: Rajesh Karyakarte

\begin{abstract}
There has been a rise in opportunistic fungal infections in the recent years. Respiratory fungal infections are an important cause for mortality and morbidity in such cases. This study was planned to study the mycological profile of respiratory samples in a tertiary care hospital. This was a retrospective study conducted over a period of 2 years in the department of Microbiology in BJGMC and Sassoon hospital, Pune. The samples were first observed under $\mathrm{KOH}$ mount and inoculated on SDA. The growth was identified using standard microbiological methods. A total of 70 respiratory samples were evaluated in the study out of which 40 were culture positive. Candida albicans was identified as the predominant species followed by Aspergillus spp, non albicans Candida and Geotrichum spp. Early diagnosis and proper understanding of the causative agent plays an important role to improve the outcome of the patients.
\end{abstract}

Keywords- Respiratory, Candida, Aspergillus

\section{INTRODUCTION}

Recent years have seen an increase in opportunistic fungal infections in immuno-compromised patients.

Respiratory fungal infections are important cause for mortality and morbidity in these patients. ${ }^{[2]}$ The species isolated, their incidence, clinical pattern, and antifungal susceptibility has also altered significantly. [3] Hence, early diagnosis, proper understanding about agents and host factors involved will help the clinician to improve the outcome of these patients. Thus, this study was planned to study the mycological profile of respiratory samples in a tertiary care hospital and to identify the predisposing factors and host factors associated with it.

\section{MATERIALS AND METHOD}

This was a retrospective laboratorybased study conducted over a period of 2 years (January 2019 to December 2020), in the department of Microbiology in BJGMC and Sassoon hospital, Pune. All the respiratory samples like sputum, bronchial alveolar lavage (BAL), endo tracheal secretions and pleural fluid which were received in mycology laboratory were included in this study. BAL, pleural fluid, endotracheal secretions were concentrated by centrifugation at $1500-2000 \mathrm{~g}$ for 5 minutes. Samples which contained clot and membranous materials. Sputum samples were treated with $\mathrm{N}$ Acetyl Cysteine prior to inoculation on Sabouraud Dextrose Agar (SDA).

All samples were first observed under $\mathrm{KOH}$ mount (20\%) and then 

India.

inoculated on 2 sets of (SDA) with and without cycloheximide was observed on at $25^{\circ} \mathrm{C}$ and $37^{\circ} \mathrm{C}$ alternate day for first week and after weekly for 4 weeks.

Growth was isolated and identified by using standard microbiological methods. Yeast identification included grams staining, germ tube test, CHROM agar identification and cornmeal agar (CMA) identification techniques. Moulds were identified using colony morphology, microscopy Lactophenol Cotton Blue (LPCB) and slide culture techniques.

Statistical Analysis- All statistical analysis was done using MS Excel.

\section{RESULTS}

A total of 70 respiratory samples were evaluated under this study. Out of 70 respiratory samples, sputum $30(42.8 \%)$ was most common followed by BAL 26 (37.14\%), Endo tracheal secretions 12 (17.1\%) and pleural fluid $2(2.86 \%)$.(Fig1)

Out of $70,40(57.1 \%)$ were culture positive. The most common age group affected was 30 to 50 years. Males were more commonly affected with ratio 1.6:1. Predisposing factors included tuberculosis (15 cases; 37.5\%), chronic obstructive pulmonary disease (12 case; 30\%), pneumonia-like lesions and lung infiltrations (10 cases; 25\%), and bronchiectasis (5 cases; 12.5\%).(Fig2). Majority of patients had outdoor occupation (farmers, laborers etc.)

Isolation rates from sputum were highest (26 samples; 65\%), followed by BAL (9 samples; 22.5\%), endo-tracheal secretions (4 samples; 10\%) and pleural fluid (2 samples; 5\%). A total of 32 samples were both $\mathrm{KOH}$ and culture positive but six sputum samples and two BAL samples were positive only by culture method.

In sputum samples, Candida albicans (15 isolates; 57.69\%) was most isolated followed by Aspergillus spp (eight isolates; $30.77 \%$ ), non-albicans candida (three isolates; 11.54\%), and Geotrichum spp (one isolate; $3.85 \%$ ). In BAL, Candida albicans (four isolates; 44.44\%), Aspergillus spp (two isolates; 22.22\%), Rhizopus (one isolate; $11.11 \%$ ) and non-albicans candida (one isolate; $11.11 \%$ ) were isolated. Endotracheal secretions was positive for Candida albicans (two isolates; 50\%) and nonalbicans candida (two isolates; 50\%).

Over-all in respiratory samples, Candida albicans (22 isolates; 55\%) was identified as the predominant species followed by Aspergillus spp (eight isolates; $20 \%$; six isolates of A. fumigatus and two isolates of $A$. flavus). Other isolates were non-albicans Candida (six isolates; 15\%), followed by Rhizopus spp (one isolate; $2.5 \%$ ) and Geotrichum spp (one isolate; $2.5 \%)$.

\section{Sample distribution}

$\square$ SPUTUM $\square$ BAL $\square$ ETT $\square$ PLEURALFLUID

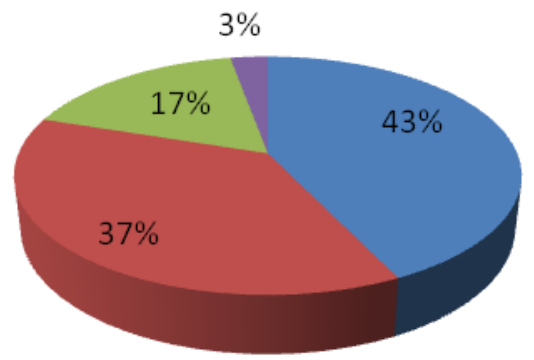

Fig:1: Sample Distribution

\section{Predisposing factors}

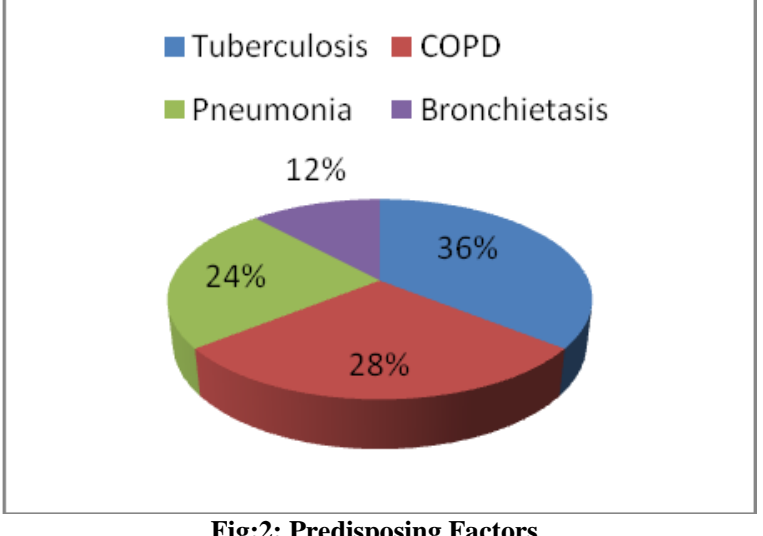

Fig:2: Predisposing Factors 

India.

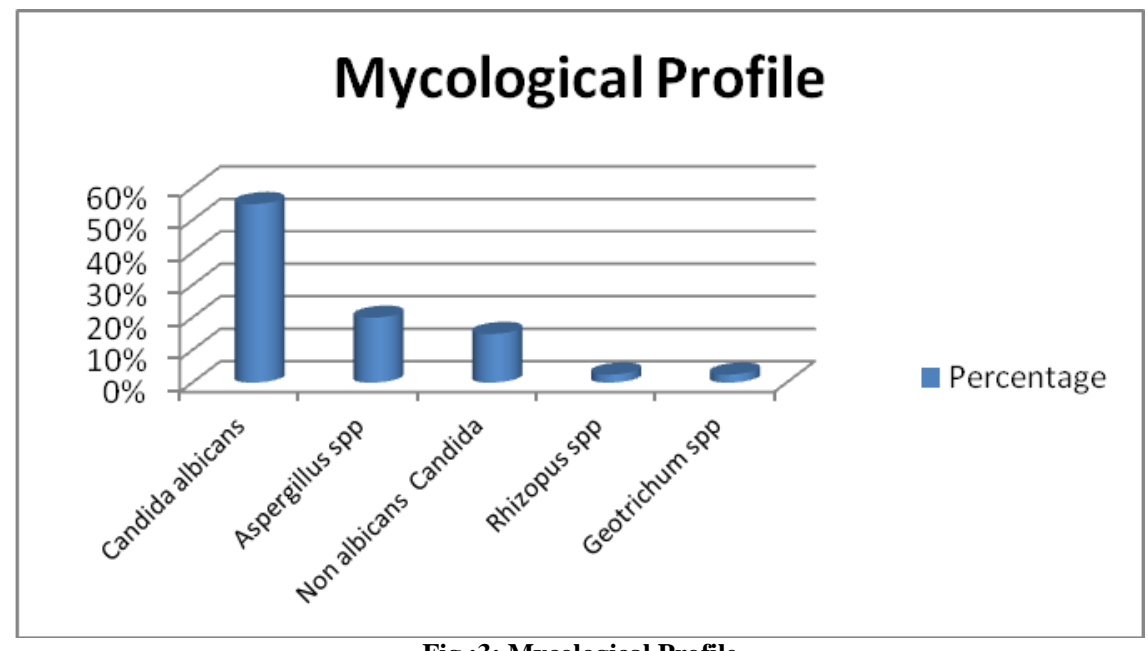

Fig :3: Mycological Profile

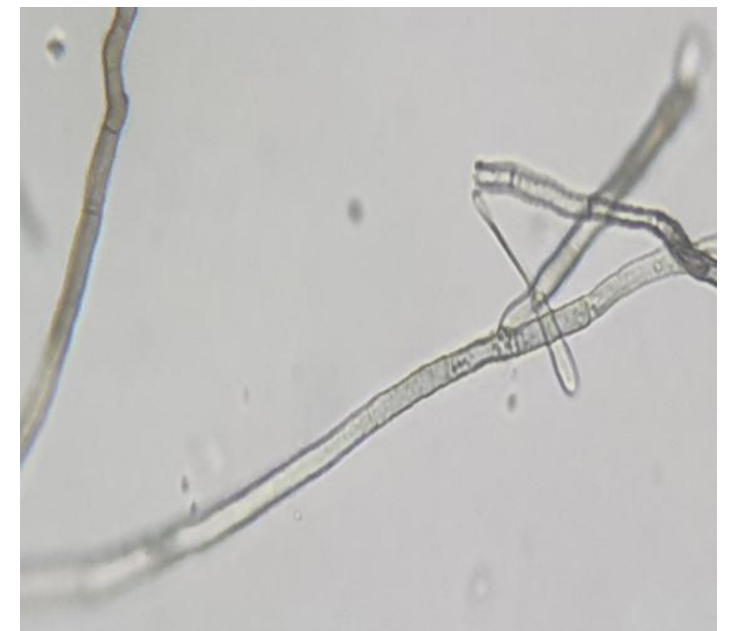

Fig 4 : KOH -Fungi filaments

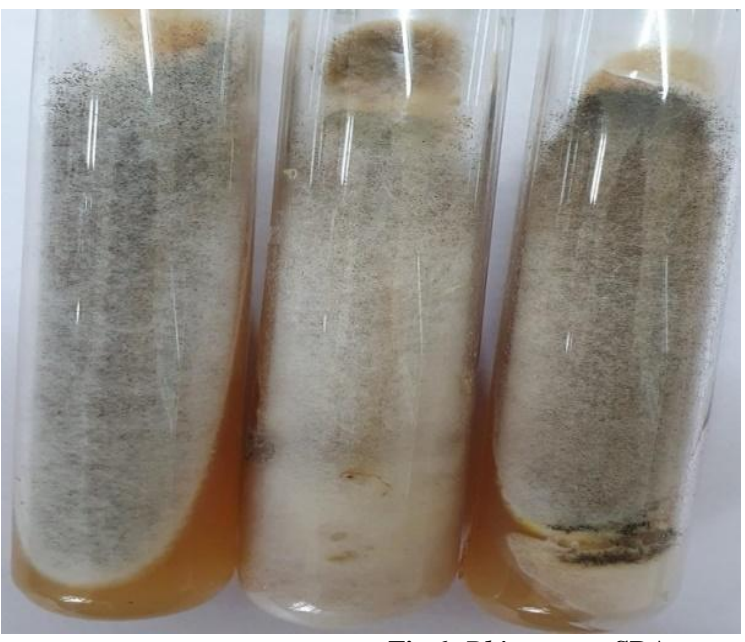

Fig 6: Rhizopus on SDA

\section{DISCUSSION}

Respiratory tract infections are important causes for mortality and morbidity in immune-compromised patients. Opportunistic fungal infections are common cause for fungal pneumoniae in developing

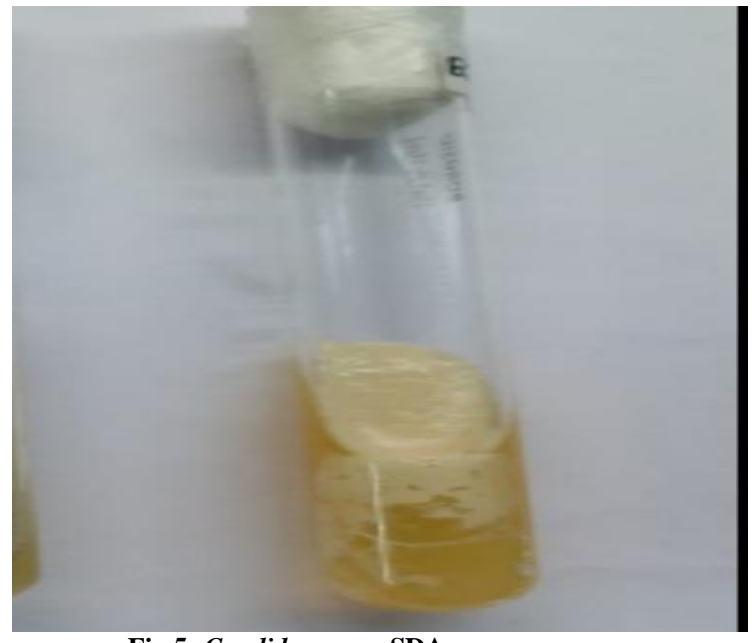

Fig 5: Candida spp on SDA

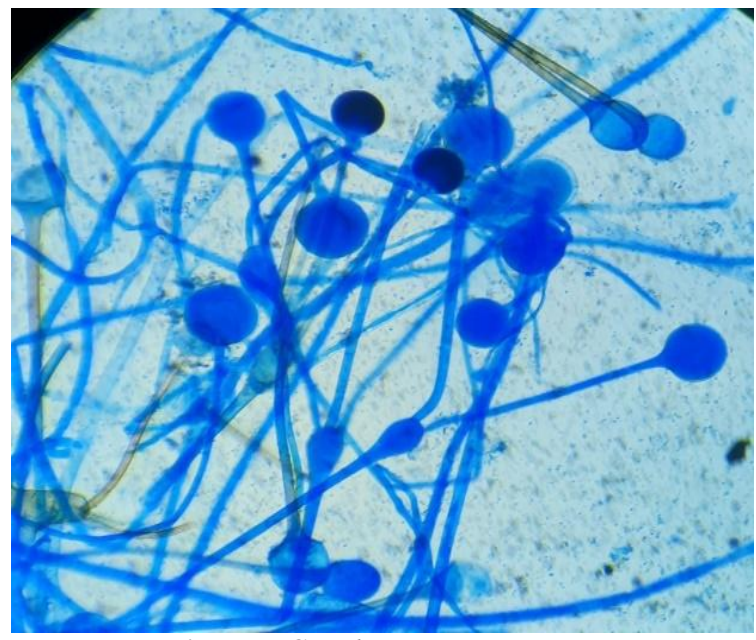

Fig 7 : LPCB $R h$

countries like India. ${ }^{[4]}$ Hence, identification of etiological fungal pathogens it is especially important for early management and treatment of patients.

In this study, $57.1 \%$ of respiratory samples were positive on fungal culture and 
sputum samples $(65 \%)$ showed the highest positivity among all samples. This was similar to the study conducted by Roohani et al. ${ }^{[5]}$ where sputum samples showed $58 \%$ positivity. It is important to note that this increase can also be due to commensal yeast and environmental inhalation of spores, which gives false positive results in sputum samples. ${ }^{[6]}$

In the present study, males were more affected which is like many other studies related to respiratory tract infections. [6,7,8] Tuberculosis is a major risk factor according to our study, which is in concordance with a study conducted by Amiri et al., in 2017. ${ }^{\text {[9] }}$

$\mathrm{KOH}$ preparation serves as a good screening test for determining the presence of fungal infections. ${ }^{[10]}$ Direct $\mathrm{KOH}$ examination helps the clinician to start early treatment and management. In present study, $80 \%$ of positive samples showed fungal filaments under $\mathrm{KOH}$ mount which helped to improve the outcome of patients due to earlier institution of appropriate treatment.

Candida albicans (55\%) was the most predominant species isolated from all respiratory samples similar to study by Kandati et al. ${ }^{[11]}$ candida species were most commonly isolated fungal pathogens. This is in contrast with a study conducted in northern India by Sharooq et al. ${ }^{6}$ where they identified Aspergillus species as major pathogen in respiratory samples.

Aspergillus species primarily affects the lungs causing upper and lower airway disorders. $^{[12,13]}$ Its spores are ubiquitous and present almost everywhere in the human environment, outdoor and indoor. ${ }^{[14]}$ Among Aspergillus species, A. fumigatus was most commonly isolated followed by A. flavus in our study, this finding is similar to Farooq $\mathrm{S}$ et al related to mycological profile of LRTI. [6]

Literature search suggests that there are only a very few studies related to mycological aspects of Respiratory samples. Fungal respiratory infections are increasing daily, hence more researches related to this has to be done for developing faster diagnostic techniques for better management of patients.

\section{CONCLUSION}

Respiratory fungal infections remain a significant cause for morbidity and mortality in the World. This study provides a basis for early detection of fungal etiology in respiratory tract infections. Early diagnosis, proper understanding of agent and host factors involved, plays an important role to improve the outcome of these patients.

\section{Acknowledgement: None}

\section{Conflict of Interest: None}

\section{Source of Funding: None}

\section{Ethical Approval: Approved}

\section{REFERENCES}

1. Badiee P, Hashemizadeh Z. Opportunistic invasive fungal infections: diagnosis \& clinical management. Indian J Med Res. 2014 Feb;139(2):195-204.

2. Li Z, Lu G, Meng G. Pathogenic Fungal Infection in the Lung. Front Immunol. 2019 Jul 3;10:1524. doi: 10.3389/fimmu.2019. 01524

3. Singh N. Trends in the epidemiology of opportunistic fungal infections: predisposing factors and the impact of antimicrobial use practices. Clin Infect Dis. 2001 Nov 15;33(10):1692-6. doi: 10.1086/323895. Epub 2001 Oct 12. PMID: 11641825.

4. Fungal Pneumonia: Overview of Fungal Pneumonia, Risk Factors, Epidemiology of Fungal Pneumonia https://emedicine.medscape.com/article/300 341-overview. Accessed December 21, 2018.

5. Roohani AH, Fatima N, Shameem M, Khan HM, Khan PA, Akhtar A. Comparing the profile of respiratory fungal pathogens amongst immunocompetent and immunocompromised hosts, their susceptibility pattern and correlation of various opportunistic respiratory fungal infections and their progression in relation 

India.

to the CD4+T-cell counts. Indian $\mathrm{J}$ Med Microbiol. 2018; 36: 408-415.

6. Farooq S, Farooq T, Dar K. Mycological profile of lower respiratory tract samples- a study from north India.IJSR.2020:9(1)

7. Supriya CS, Banu T, et al. Mycological profile of bronchial wash specimen in patients with lower respiratory tract infections. Int.J.Curr.Microbiol. App.Sci. 6(11)176-182.

8. Chandwani J, Vyas N, Hooja S, Sharma B, Maheshwari R. Mycological Profile of Sputum of HIV Positive Patients with Lower Respiratory Tract Infection and its Correlation with CD4+ T Lymphocyte Count. J Clin Diagn Res. 2016 Sep;10(9): DC28-DC31.

9. Amiri MRJ, Siami R, Khaledi A. Tuberculosis Status and Coinfection of Pulmonary Fungal Infections in Patients Referred to Reference Laboratory of Health Centers Ghaemshahr City during 20072017. Ethiop J Health Sci. 2018 Nov;28(6): 683-690.

10. Levitt JO, Levitt BH, Akhavan A, Yanofsky $H$. The sensitivity and specificity of potassium hydroxide smear and fungal culture relative to clinical assessment in the evaluation of tinea pedis: a pooled analysis. Dermatol Res Pract. 2010;2010:764843.

11. Kandati, J., Boorsu, S., Ponugoti, M., \& Samudrala,.Bacterial and fungal agents causing lower respiratory tract infections in patients with human immunodeficiency virus infection. IJRM. 4(8), 3595-3600.

12. Blanco JL, Garcia ME. Immune response to fungal infections. Vet Immunol Immunopathol. 2008 Sep 15;125(1-2):4770.

13. Barac A, Ong DSY, Jovancevic L, Peric A, Surda P, Tomic Spiric V, Rubino S. FungiInduced Upper and Lower Respiratory Tract Allergic Diseases: One Entity. Front Microbiol. 2018 Apr 3;9:583.

14. Woolnough $\mathrm{K}$, Fairs A, Pashley $\mathrm{CH}$, Wardlaw AJ. Allergic fungal airway disease: pathophysiologic and diagnostic considerations. Curr Opin Pulm Med. 2015 Jan; 21(1):39-47.

How to cite this article: Jayaram A, Sareen K, Dedwal A et.al. Mycological profile of respiratory tract samples in a tertiary care hospital from Western India. Int $J$ Health Sci Res. 2021; 11(8):1-5. DOI: https://doi.org/10. 52403/ijhsr.20210801 